\title{
Detecting Deception Using Neurofuzzy Approach
}

\author{
Otasowie Owolafe \\ Cybersecurity Science Department, Federal University of Technology \\ Nigeria
}

\begin{abstract}
The major concern of all law enforcement agencies over the years has been security. One way of arresting this concern is to detect deception consistently. Detecting deception remains a difficult task as no perfect method has been found for the detection. The main difficulty lies in the fact that no single nonverbal, verbal, or physiological response is uniquely associated with deception. In other words, the equivalent of Pinocchio's growing nose does not exist. Though detecting deception remains hard, investigators increase the odds for success by learning a few basic nonverbal (psychological) and verbal (speech) cues of deception. From past researches, single cue (verbal or nonverbal) was used; it was found that examining collection of these cues was more reliable indicator of deception than examining a single cue. Since no single verbal or non-verbal cue is able to successfully detect deception this research proposes to use both the verbal and non-verbal cues to detect deception. Therefore, this research aims to develop a neurofuzzy model for classifying extracted verbal and nonverbal features as deceptive or truthful. The system extracted desired features from the dataset of Perez-Rosas. The verbal cues capture the speech of the suspect while the nonverbal cues capture the facial expressions of the suspect. The verbal cues include the voice pitch (in terms of variations), frequency perturbation also known as jitters, pauses (voice or silent), and speechrate (is defined as the rate at which the suspect is speaking). The PRAAT (a tool for speech analysis) was used in extracting all the verbal cues. The nonverbal features were extracted using the Active Shape Model (ASM). The work was implemented using python and MatLab 2015a. The classification was done using Neurofuzzy model and the performance of the classifier on each dataset was carried out. Neurofuzzy recorded the best performance with the Nonverbal dataset (percentage score of 97.1\%) At the end of the comparative analysis it was discovered that Neurofuzzy model work well on Nonverbal dataset to detect deception. The result obtained using only verbal cue was $84.3 \%$ while that of nonverbal cue was $97.1 \%$ but on VerbNon cue it yielded $92.5 \%$ which is far better than the chance level of 50\%.
\end{abstract}

\section{Introduction}

Deception, an everyday occurrence is as old as life itself (Owolafe et al., 2018). It had its origin in the Garden of Eden when Eve was deceived by the serpent. Different behaviours are associated with deception like the eye blinking, lips movement, raised pitch, leg movement and the likes. The crucial question is to which behaviours attention should be paid. This question is difficult to answer, as research has shown that deception itself is not related to a unique pattern of specific behaviours [5], [6], [19], [23]. Detecting deception remains a difficult task [13] as no perfect method has been found for the detection [5]. In fact, multiple studies have established that lie detection results in a 50/50 chance even for experienced investigators. Although detecting deception remains difficult, investigators increase the odds for success by learning a few basic nonverbal (psychological) and verbal (speech) cues of deception. A study found that lying takes longer than telling the truth, and thus the time to answer a question may be used as a method of lie detection. However, it has also been shown that instant answers can be proof of a prepared lie. The only compromise is to try to surprise the victim and find a midway answer, not too quick, nor too long (Newman et al., 2003).

Repeated studies have shown that traditional methods of detecting deception during interviews succeed only $50 \%$ of the time, even for experienced law enforcement officers. In spite of this, investigators still need the ability to test the veracity of those they interview. To do so, investigators require a model that incorporates research with empirical experience to differentiate honesty from deception. They can use an alternative paradigm for detecting deception based on four critical domains: comfort/discomfort, emphasis, synchrony, and perception management rather than merely trying to detect traditional signs of deception, which, in some cases, may be misleading.

In real life problems are solved by thinking about them, therefore, dealing with the emulation of human thought by a computer program becomes paramount. Since humans do not think about problems as conventional computers do, dealing constantly with uncertainties, ambiguities, and contradictions arises. Sometimes deductive logic is used, but more often we think intuitively, assembling information relevant to a problem, scanning it and coming to a conclusion. Besides this, humans often learn from experience but in many ways, computers could be better at detecting deceptions than people because of their tremendous logical analysis capability and the fact 
that the logical processes used by computers are quite different from the processes used by people.

\section{Review of Related Works}

Neuro-Fuzzy Systems (NFS) are approaches where NNs are used to provide inputs for a Fuzzy System, or to change the output of a Fuzzy System to remark that the parameters of a Fuzzy System are not changed by a learning process in these approaches. If the creation of an $\mathrm{NN}$ is the main target, it is possible to apply fuzzy techniques to speed up the learning process, or to fuzzify an NN by the extension principle to be able to process fuzzy inputs. These approaches could be called Fuzzy Neural Networks to stress that fuzzy techniques are used to create or enhance NNs.

NFSs can be considered as a technique to derive a Fuzzy System from data, or to enhance it by learning from examples. In Brown and Harris [29], it was shown to be possible to use an NN to learn certain parameters of a Fuzzy System, like using a selforganizing feature map to find fuzzy rules (cooperative models), or to view a Fuzzy System as a special NN, and directly apply a learning algorithm [30] which forms the hybrid models.

The incorporation of the concept of fuzzy logic into neural network will enable a system to deal with cognitive uncertainties in a manner more like humans. The resulting hybrid system is called fuzzy neural, neural fuzzy, Neuro-fuzzy or fuzzy-neuro network. Neural networks are used to tune membership functions of fuzzy systems that are employed as decision-making systems for constructability evaluation. Although fuzzy logic can encode expert knowledge directly using rules with linguistic labels, it usually takes a lot of time to design and tune the membership functions, which quantitatively define these linguistic labels. Neural network learning techniques can automate this process and substantially reduce development time and cost while improving performance [22]. Hence, in hybrid form they can provide a perfect platform to take into account changing knowledge.

Udoh [21] presented an adaptive neuro-fuzzy discrete event system specification for monitoring petroleum products pipeline. The research was motivated by the need to have a system that can handle imprecise knowledge, learn from previous data and time oil spillage induce parametersproperties that were lacking from existing systems. In the system design, ANFIS model based on Takagi Sugeno inference mechanism was hybridized with the DEVS model based on set theory to form the DEVS-ANFIS model. The system was tested using data from Pipeline and Products Marketing Company (PPMC).
Malkawi and Murad [32] worked on artificial neuro fuzzy logic system for detecting human emotions. In the research, six models were built using different types of input/output membership functions and trained with different input arrays. The models were compared based on their ability to train with lowest error values. ANFIS editor in MATLAB was used to build the models.

Detecting deception has been a goal of humankind for centuries [24] and still presents a challenge that both researchers and practitioners are trying to meet.

DePaulo [5] stated that the reason why even motivated people fail to catch liars is because lie detection is difficult. Perhaps the main difficulty is that not a single nonverbal, verbal, or physiological response is uniquely associated with deception. In other words, the equivalent of Pinocchio's growing nose does not exist. This means that there is no single response that the lie detector can truly be relied upon.

Another difficulty is that liars who are motivated to avoid being caught may attempt to exhibit nonverbal, verbal, or physiological responses that they believe make an honest impression on lie detectors and as such, liars who employ such so-called countermeasures can indeed often fool professional lie detectors.

Meservy et al. [31] research initiative was based on behavioural approach to deception detection where they attempted to build an automated system that can infer deception or truthfulness from a set of features extracted from head and hands movements in a video. Their model, an automated unobtrusive system that identifies behavioural patterns which indicate deception from nonverbal behavioural cues and classifies deception and truth more accurately than many humans was developed.

From a communications perspective, Buller and Burgoon [4] argued that to predict the behaviour of deceivers, it is important to consider not just individual psychological variables such as motivations and emotions but also interpersonal communicative processes. They also noted that when people are trying to deceive, they are engaged in several tasks simultaneously.

DePaulo et al. [5] in their work asserted that liars form a self-presentational perspective that is they attempt to control not just their behaviours (e.g., Zuckerman et al. [23]) but also their thoughts and feelings. They also stated that liars are predicted to be less forthcoming than truth tellers (they will respond less, and in less detail, and they will seem to be holding back).

Navarro and Schafer [13] in their study found that people unwittingly signal deception via nonverbal and verbal cues. Unfortunately, no particular nonverbal or verbal cue evinces deception. 
Investigators $^{\text {ee }}$ abilities to detect deceptive behaviour depend largely on their ability to observe, catalogue, and differentiate human behaviour.

Sporer and Schwandt [26], [27] stated that deception could be detected by observing non-verbal behaviour such as body language and vocal pitch.

Vrij [19] found that examining a "cluster" of these cues was significantly more reliable indicator of deception than examining a single cue.

Dong et al. [28] developed a Neuro-fuzzy model to classify data collected from synchronous computer-mediated communication as deceptive or truthful.

Since no single verbal or non-verbal cues is able to successfully detect deception (based on the literatures reviewed), the research proposes to use both the verbal and non-verbal cues to detect deception.

\section{Methodology}

The system extracted desired features from the dataset of Perez-Rosas et al. [14]. The dataset consists of real-life trial videos of statements made by exonerees after exoneration and a few statements from defendants during crime-related TV episodes. The speakers in the videos are either defendants or witnesses. The video clips are labelled as deceptive or truthful based on a guilty verdict, not-guilty verdict, and exoneration. The dataset consists of 121 videos including 61 deceptive and 60 truthful trial clips. The average length of the videos in the dataset is 28.0 seconds. The average video length is 27.7 seconds and 28.3 seconds for the deceptive and truthful clips, respectively. The system was designed using the neurofuzzy technique. In the research, two deceptive cues were used for detecting deception. They are Verbal and Non-verbal cues. The verbal cues capture the speech of the suspect while the nonverbal cues capture the facial expressions of the suspect. The verbal cues include the voice pitch (in terms of variations), frequency perturbation also known as jitters, pauses (voice or silent), and speechrate (is defined as the rate at which the suspect is speaking). The PRAAT (a tool for speech analysis) was used in extracting all the verbal cues.

For extracting the Pitch feature, PRAAT uses the autocorrelation algorithm as shown in equation 1.

$$
r_{x}(\tau) \approx r_{x, w}(\tau) / r_{w}(\tau)
$$

Where $r_{x}(\tau)$ represent autocorrelation of the original signal, $r_{x, w}(\tau)$ is the autocorrelation of the windowed signal and $r_{w}(\tau)_{\text {is }}$ the autocorrelation of the window.

For the jitter extraction, the algorithm used is presented in equation 2 .

$$
\text { jitter }=\frac{100}{(N-1) \bar{\alpha}} \sum_{i=2}^{N}\left|\alpha_{i}-\alpha_{i-1}\right|
$$

$\alpha_{i}$ is the fundamental frequency

The pause will be extracted using equation 3 .

$$
P_{a}=T_{t}-P_{t}
$$

Where $P_{a}$ is the total number of Pauses, $T_{t}$ is Total length of time taken for the suspect to talk, $P_{t}$ is the phonetic time (actual time taken to talk).

The speechrate is extracted using equation 4 .

$$
S_{r}=N_{s} / T_{t} \quad 4
$$

Where speechrate is denoted as $S_{r}$, number of syllabus as $N_{s}$, and total time taken as $T_{t}$.

The nonverbal cues (that is the facial expressions) to be extracted using the Active Shape Model (ASM) are: Eyelid Blinking, Lip movement, eyebrown movement. To form the shape model, lot of training examples (in this case, different faces) were collected and the correspondence for each of the training examples were formed. Consider a person (a face), $\mathrm{j}$ from the set of training examples, the $n^{\text {th }}$ feature points of the person $\mathrm{j}$ and for all the training set is given by equation 5 .

$z^{j}=\left\{\left(x_{1}^{j}, y_{1}^{j}\right),\left(x_{2}^{j}, y_{2}^{j}\right),\left(x_{3}^{j}, y_{3}^{j}\right) \ldots\left(x_{n}^{j}, y_{n}^{j}\right)\right\}$.

Since all the shapes may not be properly aligned, the shapes are rotated and translated to be centred at the origin $(0,0)$. After translation, the dimension of the set of aligned shapes is reduced using PCA. Any shape can then be approximated using equation 6 .

$$
Z=\mu+P b
$$

where $\mathrm{b}$ is the model parameters, $P=V_{1}, V_{2} \ldots V_{k}$.

The next step after the feature extraction stage is the neurofuzzy model design. The model has 7 inputs (corresponding to the extracted features) each having 3 membership functions corresponding mostly to low, normal and high. The neurofuzzy architecture consists of five layers. The first and the fourth layers are known as the adaptive nodes since they have parameters to be learnt, while the second, third and fifth layers are fixed nodes since they contain no learning parameters. The output of one layer is used as input in subsequent layer. Layer 5 computes the overall output as the summation of all incoming signals as given in equation 7 .

$$
O_{i}^{5}=\sum_{i=1}^{n} \bar{w}_{i} f_{i}=\frac{\sum_{i=1}^{n} w_{i} f_{i}}{\sum_{i=1}^{n} w_{i}}
$$

After the neurofuzzy model design, features extracted from videos with known classification were used in training the designed model in other to 
form a baseline. The trained system was then used to classify videos whose classification is unknown.

\subsection{System Architecture}

The system architecture is as presented in Figure 1.

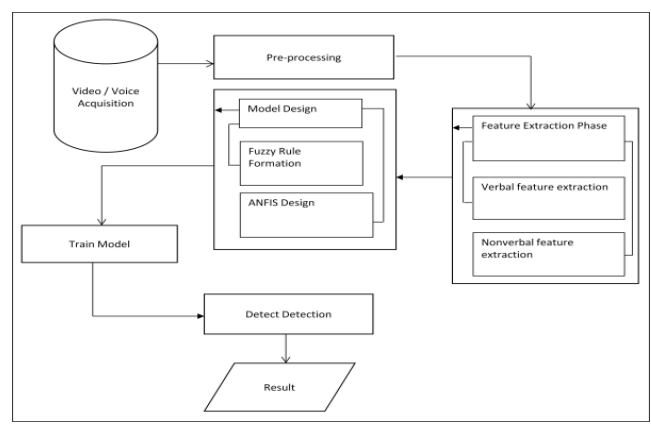

Figure 1. Deception Detection Architecture

3.2.1 Video and Voice Acquisition. Getting the deceptive and non-deceptive data is an essential part of the research. Previous researches made use of staged scenario where the participants were asked to either lie or tell the truth for some gains. In this research, the participants were either the defendants or witnesses that have something at stake, some of the participants were not aware of the presence of a video capturing device. The data used for this research can be termed high stake data since the participants were not asked to lie or tell the truth for some rewards. The difference between the current research and that of other researchers is that the participants in this research were not asked to lie or tell the truth, they were allowed to answer the questions based on their perception and understanding of the questions asked.

3.2.2. Preprocessing. The analysis or recognition of a shape is often perturbed by noise, thus the smoothing of object boundaries is a necessary preprocessing step. Also, when zooming or warping binary digital images, one obtains a result that must be smoothed for better visualization. The smoothing procedure can also be used to extract some shape characteristics: by making the difference between the original and the smoothed object, salient or carved parts can be detected and measured.

The filter object in the PRAAT window was used in removing noise from the voice data. The range of the filter frequency, smoothing bandwidth was set to the desired values also the spectral subtraction was used for noise reduction.

3.2.3. Feature Extraction. Features are the characteristics of the objects of interest or salient features in an image. Feature extraction is the technique of extracting these salient features from images of different abnormal categories in such a way that class similarity is either minimized or maximized.

In classification problem, the use of salient features is essential for accuracy according to Hermosilla et al. [25]. The use of a model that can fit the shape of the image of interest from the dataset becomes paramount. The Active Shape Model (ASM) is a model that can deform to fit the shape of the image of interest. The verbal and the nonverbal cues data will be extracted from a sufficient voice and video database. Since there is no ready-to-use database for this research, a data collection was designed and set up to create the speech and video database.

\section{a) Nonverbal Cues and Features Extraction}

Nonverbal cues are leakages or deformations that occur in the body channels of the person being interrogated or interviewed. The nonverbal cues considered in this research are:

- Eye brown movement: the element in the Eye brown movement set are \{Eye brown_raised, eye brown_normal, eyebrown_lowered

- Eyelid movement (interval between each twitch): Eye blink is a quick action of closing and opening of the eyelids (Le et al., 2013). A blink's duration is defined as the count of consecutive frames of closure. The elements in the eyelid blinking set are \{Eyelid closed, slightly opened, widely opened $\}$.

- Lip movement: the elements in the lip set are $\{$ Lip_raised, lip_normal, and

lip_protruded

Nose movement: the elements in the nose set are \{nose_raised, nose_normal, and nose_enlarged .

Table 1. Degree of Membership for nonverbal Cues

\begin{tabular}{|r|c|lc|}
\hline \multicolumn{1}{|c|}{$\mathrm{S}$} & \multicolumn{2}{|c|}{ Linguistic } & \multicolumn{2}{|c|}{ Normal Range } \\
/N & Variables & Min & Max \\
\hline 1 & $\begin{array}{c}\text { Eyebrown } \\
\text { movement }\end{array}$ & 10 & 50 \\
\hline 2 & $\begin{array}{c}\text { Eyelid } \\
\text { blinking }\end{array}$ & 20 & 70 \\
\hline 3 & $\begin{array}{c}\text { Lip } \\
\text { movement }\end{array}$ & 10 & 50 \\
\hline 4 & Nose & 20 & 70 \\
\hline
\end{tabular}

\section{b) Fuzzification and Membership Functions}

In fuzzy inference system, fuzzification is the first point of call. In real word, most of the variables are crisp in nature. Therefore, there is need to convert the crisp variables (both input and output) to fuzzy variables and then apply fuzzy inference to process those data to obtain the desired output. There are two steps in fuzzification process: derive the membership functions for input and output variables and represent 
them with linguistic variables. In practice, membership functions can have different types, such as the triangular form, trapezoidal form, Gaussian form and the bell-shaped form. A linguistic expression from the natural language can be used to label the fuzzy sets in order to express their semantics. This construct is essential in the fuzzy logic theory and is called a linguistic variable. A linguistic variable is a variable whose values are words or sentences instead of numerical values. These values are called terms (also linguistic or verbal terms) and are represented by fuzzy sets.

Pause: three linguistic variables used to represent pauses are: low (200-220ms), normal (210-240ms) and high (230-250ms). The membership function graph is shown in figure 3.5 while the degree of membership for low, normal and high are:

$$
P_{a}= \begin{cases}\text { Low } & \text { if } x<220 \\ \text { Normal } & \text { if } 210 \leq x<240 \\ \text { High } & \text { if } x>230 \\ \mu_{\text {low }}(x)=\{0,0.5,1.0\} \\ \mu_{\text {nor }}(x)=\{0.5,1.0,1.5\} \\ \mu_{\text {high }}(x)=\{1,1.5,2.0\}\end{cases}
$$$$
\text { Low }\left(P_{a}\right)=\left\{\begin{array}{cc}
0 & \text { if } x<0 \\
\frac{x}{0.5} & \text { if } 0 \leq x<0.5 \\
1 & \text { if } x \geq 0.5
\end{array}\right.
$$$$
\operatorname{Normal}\left(P_{a}\right)=\left\{\begin{array}{cc}
0 & \text { if } x<0.5 \\
\frac{x-0.5}{0.5} & \text { if } 0.5 \leq x<1.0 \\
1 & \text { if } x \geq 1.0
\end{array}\right.
$$$$
\operatorname{High}\left(P_{a}\right)= \begin{cases}0 & \text { if } x<1.0 \\ \frac{x-1.0}{0.5} & \text { if } 1.0 \leq x<1.5 \\ & \text { if } x \geq 1.5 \\ 1 & \end{cases}
$$

Speechrate: three linguistic variables used to represent Speechrate are: low (133-160wps), normal (147-174wps) and high (166-188wps). The degree of membership for low, normal and high are:

$$
S_{r}=\left\{\begin{array}{lc}
\text { Low } & \text { if } x<160 \\
\text { Normal } & \text { if } 147 \leq x<174 \\
\text { High } \quad & \text { if } x>166 \\
\mu_{\text {low }}(x)=\{0,0.5,1.0\} \\
\mu_{\text {nor }}(x)=\{0.5,1.0,1.5\} \\
\mu_{\text {high }}(x)=\{1,1.5,2.0\}
\end{array}\right.
$$

Low $\left(S_{r}\right)=\left\{\begin{array}{cl}0 & \text { if } x<0 \\ \frac{x}{0.5} & \text { if } 0 \leq x<0.5 \\ 1 & \text { if } x \geq 0.5\end{array}\right.$

Normal $\left(S_{r}\right)=\left\{\begin{aligned} 0 & \text { if } x<0.5 \\ \frac{x-0.5}{0.5} & \text { if } 0.5 \leq x<1.0 \\ 1 & \text { if } x \geq 1.0\end{aligned}\right.$

High $\left(S_{r}\right)=\left\{\begin{aligned} 0 & \text { if } x<1.0 \\ \frac{x-1.0}{0.5} & \text { if } 1.0 \leq x<1.5 \\ 1 & \text { if } x \geq 1.5\end{aligned}\right.$

Pitch: can assume any of the three values: minimum $(120-180 \mathrm{~Hz})$, medium $(150-210 \mathrm{~Hz})$ and maximum $(180-265 \mathrm{~Hz})$. The degree of membership for minimum, medium and maximum are:

$$
P_{t}=\left\{\begin{array}{l}
\text { Minimum if } x<180 \\
\text { Medium if } 150 \leq x<210 \\
\text { Maximum if } x>265
\end{array}\right.
$$

$$
\begin{gathered}
\mu_{\text {low }}(x)=\{0,0.5,1.0\} \\
\mu_{\text {nor }}(x)=\{0.5,1.0,1.5\} \\
\mu_{\text {high }}(x)=\{1,1.5,2.0\}
\end{gathered}
$$

Minimum $\left(P_{t}\right)=\left\{\begin{array}{cl}0 & \text { if } x<0 \\ \frac{x}{0.5} & \text { if } 0 \leq x<0.5 \\ 1 & \text { if } x \geq 0.5\end{array}\right.$
Medium $\left(P_{t}\right)=\left\{\begin{array}{cc}0 & \text { if } x<0.5 \\ \frac{x-0.5}{0.5} & \text { if } 0.5 \leq x<1.0 \\ 1 & \text { if } x \geq 1.0\end{array}\right.$
Maximum $\left(P_{t}\right)=\left\{\begin{array}{cc}0 & \text { if } x<1.0 \\ \frac{x-1.0}{0.5} & \text { if } 1.0 \leq x<1.5 \\ 1 & \text { if } x \geq 1.5\end{array}\right.$ 
Jitter: can assume any of the three values: low (9099), normal (95 - 104) and high (100-110). The degree of membership for low, normal and high are:

$$
J_{t}= \begin{cases}\text { Low } & \text { if } x<99 \\ \text { Normal } & \text { if } 95 \leq x<104 \\ \text { High } & \text { if } x>110\end{cases}
$$

$$
\begin{gathered}
\mu_{\text {low }}(x)=\{0,0.5,1.0\} \\
\mu_{\text {nor }}(x)=\{0.5,1.0,1.5\} \\
\mu_{\text {high }}(x)=\{1,1.5,2.0\}
\end{gathered}
$$

Low $\left(J_{t}\right)=\left\{\begin{array}{cl}0 & \text { if } x<0 \\ \frac{x}{5} & \text { if } 0 \leq x<0.5 \\ 1 & \text { if } x \geq 0.5\end{array}\right.$

Normal $\left(J_{t}\right)=\left\{\begin{array}{cc}0 & \text { if } x<0.5 \\ \frac{x-0.5}{0.5} & \text { if } 0.5 \leq x<1.0 \\ 1 & \text { if } x \geq 1.0\end{array}\right.$

High $\left(J_{t}\right)=\left\{\begin{array}{cl}0 & \text { if } x<1.0 \\ \frac{x-1.0}{0.5} & \text { if } 1.0 \leq x<1.5 \\ 1 & \text { if } x \geq 1.5\end{array}\right.$

\subsection{Training/Testing}

The deceptive and non-deceptive corpus developed was divided into two (one for training, the other for testing). The datasets used for training the different models is presented in Table 3.3. The system was trained using the hybrid learning algorithm approach with an error tolerance of 0.001 . Features extracted from the videos with known classification were used in training the system in other to form a truth baseline. The remaining datasets was then used to test the trained model.

Table 2. Training and Testing Datasets for all Cues

\begin{tabular}{|l|c|c|c|}
\hline & $\begin{array}{l}\text { Verbal } \\
\text { Cues }\end{array}$ & $\begin{array}{l}\text { Nonverbal } \\
\text { Cues }\end{array}$ & $\begin{array}{l}\text { VerbNon } \\
\text { Cues }\end{array}$ \\
\hline $\begin{array}{l}\text { Training } \\
\text { Dataset }\end{array}$ & 933 & 2998 & 1000 \\
\hline Test & 760 & 2136 & 353 \\
\hline Total & 1693 & 5134 & 1353 \\
\hline
\end{tabular}

\subsection{Classification}

After the model design, the verbal and nonverbal dataset was each used for testing the model. The verbal cues extracted were used in testing the designed model and the classification rate was found to be $84.3 \%$. In the nonverbal the classification rate was found to be $97.1 \%$. When both verbal and nonverbal cues were used the classification, rate was found to be $92.5 \%$.

\section{Result Testing and Evaluation}

The model was tested using dataset with known classification. Details of the analysis are shown in Table 3 and the graph is shown in Figure 4.31. Nonverbal dataset has reduced training error as well as reduce testing error.

Table 3. Training versus Testing Error across datasets

\begin{tabular}{|l|c|c|c|c|c|}
\hline & $\begin{array}{l}\text { Total } \\
\text { dataset }\end{array}$ & $\begin{array}{l}\text { Training } \\
\text { dataset }\end{array}$ & $\begin{array}{l}\text { Testing } \\
\text { dataset }\end{array}$ & $\begin{array}{l}\text { Training } \\
\text { Error }\end{array}$ & $\begin{array}{l}\text { Testing } \\
\text { Error }\end{array}$ \\
\hline Verbal & 1693 & 933 & 760 & 0.3354 & 0.8745 \\
\hline $\begin{array}{l}\text { Non } \\
\text { verbal }\end{array}$ & 5133 & 2998 & 2135 & 0.26156 & 0.5432 \\
\hline $\begin{array}{l}\text { Verb } \\
\text { Non }\end{array}$ & 1353 & 1000 & 353 & 0.15214 & 1.8580 \\
\hline
\end{tabular}

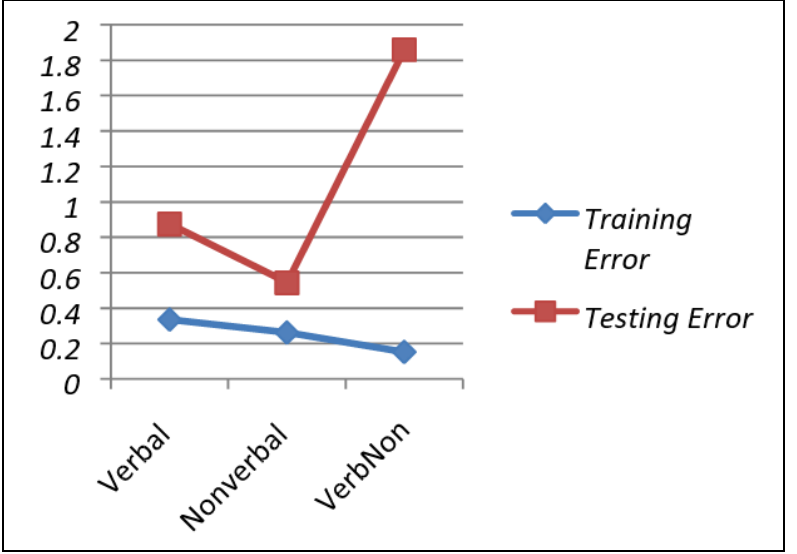

Figure 2. Training versus Testing Error across datasets

\subsection{Performance Metrices}

The metrics used for carrying out the performance evaluation are listed as: 
1. False Positive Rate (FPR):

$$
F_{N, R}=\frac{F_{P}}{T_{P}+F_{P}}
$$

2. True Positive Rate (TPR):

$$
T_{P, R}=\frac{T_{P}}{T_{P}+F_{N}}
$$

3. Accuracy: The overall accuracy is given by the sum of true and false utterances correctly classified, out of all the classifications carried out. It is the number of correct predictions over the total number of predictions.

$$
\text { Accuracy }=\sum\left(\frac{T_{P}+F_{P}}{T_{P}+T_{N}+F_{P}+F_{N}}\right)
$$

Where $T_{P}, T_{N}, F_{P}$ and $F_{N}$ are the True positive, True negative, False positive and False negative values respectively.

4. Confusion Matrix: It is a table used to describe the performance of the classification model on the dataset.

Table 4 shows the extracted confusion matrix and Accuracy for each of the datasets.

Table 4. Confusion Matrix for Verbal, Nonverbal and VerbNon dataset

\begin{tabular}{|l|c|c|c|c|}
\hline & Training & Validation & Test & All \\
\hline $\begin{array}{l}\text { Nonverbal } \\
\text { (N) }\end{array}$ & $97.1 \%$ & $97.2 \%$ & $97.2 \%$ & $97.1 \%$ \\
\hline Verbal (V) & $84.4 \%$ & $86.6 \%$ & $81.9 \%$ & $84.3 \%$ \\
\hline VerbNon & $92.7 \%$ & $92.8 \%$ & $91.6 \%$ & $92.5 \%$ \\
\hline
\end{tabular}

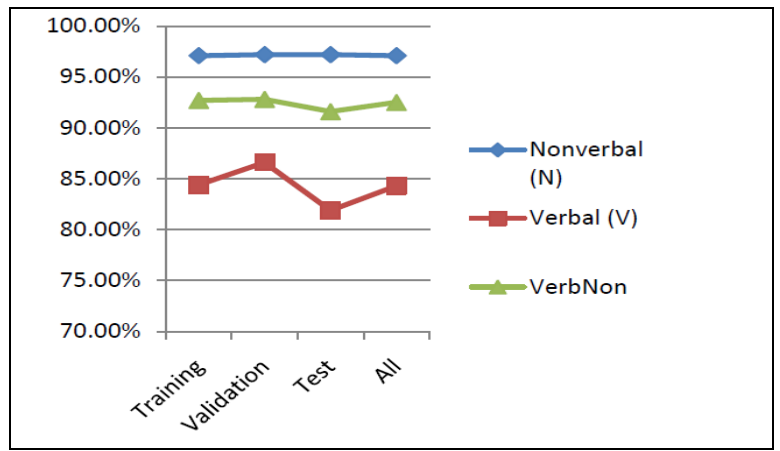

Figure 3. Confusion matrix for verbal, nonverbal and VerbNon dataset

\subsection{Analysis of Neurofuzzy classifier on Different dataset}

The different datasets were passed through the Neurofuzzy classifiers to ascertain the performance. Table 5 shows the performance of the classifier on each of the datasets while Figure 4 gives graphical representations of the performance. From the table, it is observed that Neurofuzzy recorded the best performance with the Nonverbal dataset (percentage score of $97.1 \%$ ). The result obtained using only verbal cue was $84.3 \%$ while that of nonverbal cue was $97.1 \%$ but on VerbNon it yielded $92.5 \%$.

Table 5. Comparative Analysis of Neurofuzzy classifier on Different dataset

Verbal Cues

\begin{tabular}{|l|c|}
\hline & Neurofuzzy \\
\hline Overall Accuracy & $84.3 \%$ \\
\hline Overall Error & $15.7 \%$ \\
\hline $\begin{array}{l}\text { Total Dataset } \\
\text { used }\end{array}$ & 1693 \\
\hline
\end{tabular}

Nonverbal Cues

\begin{tabular}{|l|c|}
\hline & Neurofuzzy \\
\hline Overall Accuracy & $97.1 \%$ \\
\hline Overall Error & $2.9 \%$ \\
\hline $\begin{array}{l}\text { Total Dataset } \\
\text { used }\end{array}$ & 5133 \\
\hline
\end{tabular}

\section{VerbNon Cues}

\begin{tabular}{|l|c|}
\hline & Neurofuzzy \\
\hline Overall Accuracy & $92.5 \%$ \\
\hline Overall Error & $7.5 \%$ \\
\hline $\begin{array}{l}\text { Total Dataset } \\
\text { used }\end{array}$ & 1353 \\
\hline
\end{tabular}

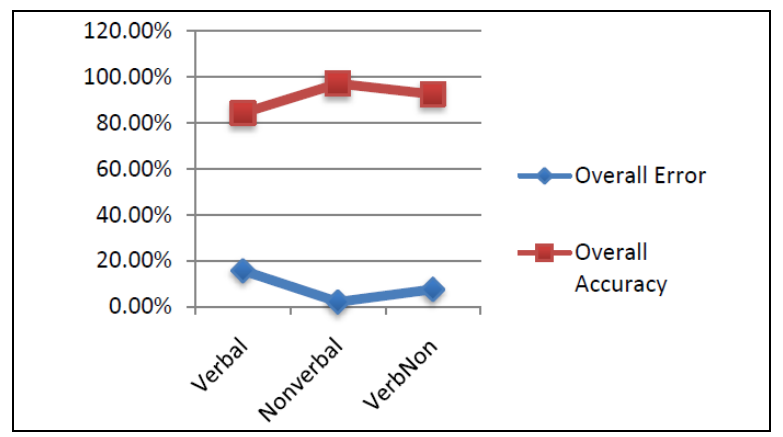

Figure 4. Accuracy and Error rate across dataset 


\section{Conclusion}

Deception detection is an involved social issue because to successfully deceive the deceiver has to formulate a story that is internally consistent while hiding emotions and true intentions. Facial expressions and voice play a critical role in the identification of deception as shown in this research. Previous research made use of only one cue, but this research made use of both verbal and nonverbal cues. The developed system (VerbNon Cue) was able to perform better than chance and trained professionals with a result difference of $42.5 \%$.

This work uses verbal, nonverbal cues and a combination of both cues to detect deception. The verbal cues were extracted using Praat while the nonverbal was extracted using Active Shape Model. The classification was done using Neurofuzzy model.

The proposed system was implemented using Matlab 2015a on window 7 with 2GB RAM. The extracted data was divided into training data and test data. The neurofuzzy model was trained using the training data while the functionality of the model was ascertained using the test data. At the end of the comparative analysis it was discovered that Neurofuzzy model work well on Nonverbal dataset to detect deception. The result obtained using only verbal cue was $84.3 \%$ while that of nonverbal cue was $97.1 \%$ but on VerbNon yielded $92.5 \%$ which is far better than the chance level of $50 \%$.

\section{References}

[1] Almela A., Valencia-Garcia R., and Cantos P., (2012). Seeing through deception: A computational approach to deceit detection in written communication. In Proceedings of the Workshop on Computational Approaches to Deception Detection, pages 15-22, Avignon, France, April.

[2] Bachenko, J., Fitzpatrick, E., Schonwetter, M., (2008). Verification and implementation of language-based deception indicators in civil and criminal narratives. In Proceedings of the 22nd International Conference on Computational Linguistics, Manchester, U.K., 18-22 August 2008, pp. 41-48.

[3] Baron-Cohen S., Wheelwright, S., Hill J., Raste Y., and Plumb, I., (2001). "The Reading the Mind in the Eyes" Test Revised Version: A Study with Normal Adults, and Adults with Asperger Syndrome or Highfunctioning Autism. Journal of Child Psychology and Psychiatry, vol. 42, no.2, pp. 241251.
[4] Buller, D.B, and Burgoon, J.K., (1996). Interpersonal Deception Theory. Communication theory, vol6, pp 203-242.

[5] DePaulo, B. M., Lindsay, J. J., Malone, B. E., Muhlenbruck, L., Charlton, K., \& Cooper, H. (2003). Cues to deception. Psychological Bulletin, 129, 74112.

[6] Ekman, P., (1985/1992). Telling lies: Clues to deceit in the marketplace, politics, and marriage (2nd ed.). New York: Norton.

[7] Enos, F., Benus, S., Cautin, R. L., Graciarena, M., Hirschberg, J. and Shriberg, E., (2006). Personality factors in human deception detection: Comparing human to machine performance. Proceedings of the 9th International Conference on Spoken Language Processing, ISCA 2006, Pittsburgh, USA

[8] Gamson, Rachel, and Jessica Gottesman, Nicholas Milan and Sitara Weerasuriya. (2012), "Cues to Catching Deception in Interviews." College Park, MD: START, 2012.

[9] Jaume M, Eugenio G, and Carmen H. (2004). Defining Deception. Journal of anales de psicologia, vol 20 no. 1, pp 147-171.

[10] Knapp, M. L., and Comadena, M. A., (1979). Telling it like it isn't: A review of theory and research on deceptive communications. Human Communication Research, 5, 270-285.

[11] Mitchell, R. W., and Thompson, N. S., (1986). Deception: Perspectives on human and nonhuman deceit. Albany: SUNY Press.

[12] Mitchell, R. W., (1986). A framework for discussing deception. In R. W. Mitchell \& N. Thomson (Eds.), Deception, perspectives on human and nonhuman deceit (pp. 3-40). New York: State University of New York Press.

[13] Navarro J and Schafer R. J (2001). Detecting Deception, FBI Law Enforcement Bulletin.

[14] Perez-Rosas V., Abouelenien M., Mihalcea R., Xiao Y., Linton C. J., Burzo M., (2015). Verbal and Nonverbal Clues for Real-life Deception Detection. In Proceedings of the 2015 Conference on Empirical Methods in Natural Language Processing, pages 2336-2346, Lisbon, Portugal, 17-21 September 2015. c 2015 Association for Computational Linguistics. 
[15] Reid J. and Associates (2000). The Reid Technique of Interviewing and Interrogation. Chicago: John E. Reid and Associates, Inc.

[16] Rothwell J., Bandar Z., O'Shea J. and McLean D., (2006). Charting the behavioural state of a person using a Backpropagation Neural Network. Journal of Neural Computing and Applications. DOI 10.1007/s00521-006-0055-9. 2006.

[17] Vrij, A., (2000). Detecting lies and deceit: The psychology of lying and its Implications for professional practice. Chichester: John Wiley.

[18] Vrij, Fisher, Mann and Leal, (2008). A Cognitive Load Approach to Lie Detection. Journal of Investigative Psychology and Offenders Profiling, Volume 5, Issue 1-2, 2008, Pages 39- 43.

[19] Vrij, A., (2008). Detecting lies and deceit: Pitfalls and opportunities. Chichester, United Kingdom: Wiley.

[20] Zhou, L., Twitchell, D.P., Qin, T., Burgoon, J.K., and Nunamaker, J.F., Jr. (2003) An exploratory study into deception detection in text-based computer-mediated communication, in Proceedings of the Thirty-Sixth Annual Hawaii.

[21] Udoh, S. S., (2016). Adaptive Neuro-Fuzzy Discrete Event System Specification for Monitoring Petroleum Products Pipeline. PhD Dissertation, Department of Computer Science, School of Sciences, Federal University of Technology, Akure, Nigeria, 178-212.

[22] Kosko, B., (1996). Neural networks and fuzzy systems, Prentice-Hall International, Englewood Cliffs, N.J., U.S.A., 3rd edition.

[23] Zuckerman, M., DePaulo, B. M., \& Rosenthal, R., (1981). Verbal and nonverbal communication of deception. In L. Berkowitz (Ed.), Advances in experimental social psychology (Vol. 14, pp. 1-59). New York: Academic Press.

[24] Granhag, P. A., \& Stroemwall, L. A., (2004). Research on deception detection: Intersections and future challenges. In P. A. Granhag \& L. A. Stroemwall (Eds.), The deception of detection in forensic contexts (pp. 317-330). Cambridge: Cambridge University Press.

[25] Hermosilla, J., delRio, P., Koennoel, A.T., (2010). Diversity of eco-innovations: reflections from selected case studies. Journal of Cleaner Production 18, 20-11, 1073-1083.
[26] Sporer, S. L., \& Schwandt, B. (2006). Paraverbal indicators of deception: A meta-analytic synthesis. Applied Cognitive Psychology, 20(4), 421-446. DOI: https://doi.org/10.1002/acp.1190

[27] Sporer, S. L., \& Schwandt, B., (2007). Moderators of nonverbal indicators of deception: A meta-analytic synthesis. Psychology, Public Policy, and Law, 13(1), 1-34. DOI: https://doi.org/10.1037/ 1076-8971.13.1.1.

[28] Dong, W., Huang, N., Ming, Y., 2008. Reliability analysis of component-based software based on relationships of components, IEEE Conference on Web Services, pp. 814-815.

[29] Brown M. \& Harris C.J., (1995). Neurofuzzy Adaptive Modelling and Control. Prentice Hall, 1st edition, Hemel Hempstead.

[30] Nomura, H., Hayashi, I., and Wakami, N., (1992). A Learning Method of Fuzzy Inference Rules by Descent Method. In Proc. IEEE Int. Conf. on Fuzzy Systems 1992, pages 203\{210, San Diego.

[31] Meservy, T., Jensen, M. L., Kruse, J., Burgoon, J. K., \& Nunamaker, J. F. (2005). Automatic Extraction of Deceptive Behavioral Cues from Video. Paper presented at the Intelligence and Security Informatics Conference, Atlanta, GA.

[32] M. Malkawi and O. Murad, (2013). "Artificial neuro fuzzy logic system for detecting human emotions", Humancentric Computing and Information Sciences 2013, vol. 3, no. 3. 